\title{
Coupling primary and stem cell-derived cardiomyocytes in an in vitro model of cardiac cell therapy
}

\section{Citation}

Aratyn-Schaus, Yvonne, Francesco S. Pasqualini, Hongyan Yuan, Megan L. McCain, George J.C. Ye, Sean P. Sheehy, Patrick H. Campbell, and Kevin Kit Parker. 2016. “Coupling primary and stem cell-derived cardiomyocytes in an in vitro model of cardiac cell therapy." The Journal of Cell Biology 212 (4): 389-397. doi:10.1083/jcb.201508026. http://dx.doi.org/10.1083/jcb.201508026.

\section{Published Version}

doi:10.1083/jcb.201508026

\section{Permanent link}

http://nrs.harvard.edu/urn-3:HUL.InstRepos:29002688

\section{Terms of Use}

This article was downloaded from Harvard University's DASH repository, and is made available under the terms and conditions applicable to Other Posted Material, as set forth at http:// nrs.harvard.edu/urn-3:HUL.InstRepos:dash.current.terms-of-use\#LAA

\section{Share Your Story}

The Harvard community has made this article openly available.

Please share how this access benefits you. Submit a story.

\section{Accessibility}




\title{
Coupling primary and stem cell-derived cardiomyocytes in an in vitro model of cardiac cell therapy
}

\author{
Yvonne Aratyn-Schaus, ${ }^{*}$ Francesco S. Pasqualini, ${ }^{*}$ Hongyan Yuan, Megan L. McCain, George J.C. Ye, Sean P. Sheehy, \\ Patrick H. Campbell, and Kevin Kit Parker
}

Disease Biophysics Group, Wyss Institute of Biologically Inspired Engineering, John A. Paulson School of Engineering and Applied Sciences, Harvard University, Cambridge, MA 02138

The efficacy of cardiac cell therapy depends on the integration of existing and newly formed cardiomyocytes. Here, we developed a minimal in vitro model of this interface by engineering two cell microtissues ( $\mu$ tissues) containing mouse cardiomyocytes, representing spared myocardium after injury, and cardiomyocytes generated from embryonic and induced pluripotent stem cells, to model newly formed cells. We demonstrated that weaker stem cell-derived myocytes coupled with stronger myocytes to support synchronous contraction, but this arrangement required focal adhesion-like structures near the cell-cell junction that degrade force transmission between cells. Moreover, we developed a computational model of $\mu$ tissue mechanics to demonstrate that a reduction in isometric tension is sufficient to impair force transmission across the cell-cell boundary. Together, our in vitro and in silico results suggest that mechanotransductive mechanisms may contribute to the modest functional benefits observed in cell-therapy studies by regulating the amount of contractile force effectively transmitted at the junction between newly formed and spared myocytes.

\section{Introduction}

Stem cell transplantation therapy has shown a positive safety outcome (Menasché et al., 2001; Strauer et al., 2002; Makkar et al., 2012) but poor (Makkar et al., 2012; Traverse et al., 2012) and inconsistent (Abdel-Latif et al., 2007; Bolli et al., 2011; Donndorf et al., 2011) improvements to heart function in clinical trials. Similarly, preclinical studies demonstrated stem cell engraftment (Kehat et al., 2004; Shiba et al., 2012) but limited contractile benefits (Kehat et al., 2004; Laflamme et al., 2007).

We reasoned that to repair the contractile properties of the heart, mechanical forces must be transmitted across the boundaries between the newly generated myocytes and spared myocardium. This entails the formation of intercalated disks, specialized cell-cell junctions that transmit electrochemical signals (Bers, 2002) and mechanical forces (Parker and Ingber, 2007). The coordinated assembly of these structures relies on the distribution and remodeling of cell-matrix and cell-cell adhesions (Wu et al., 2002; Hirschy et al., 2006; McCain et al., 2012b), which further depends on the contractile state of the cell cytoskeleton. Unfortunately, cellular traction forces between newly formed and existing myocytes cannot be measured in vivo.

*Y. Aratyn-Schaus and F.S. Pasqualini contributed equally to this paper.

Correspondence to Kevin Kit Parker: kkparker@seas.harvard.edu

Abbreviations used in this paper: mES-CM, murine embryonic stem cellderived myocyte; $\mu$ tissue, microtissue; miPS-CM, murine induced pluripotent stem cell-derived myocyte; OOP, orientational order parameter; TFM, traction force microscopy.
Our hypothesis is that newly formed myocytes exhibit weaker contractile strength that limits force transmission at the junction with primary myocytes. To test this hypothesis, we developed an in vitro assay to study the mechanical coupling between two cell microtissues ( $\mu$ tissues). As in vitro proxies for native and newly formed myocytes, we used murine neonate ventricular myocytes and immature murine embryonic stem cellderived myocytes (mES-CMs; Sheehy et al., 2014) or murine induced pluripotent stem cell-derived myocytes (miPS-CMs), respectively. Immunohistochemistry revealed aligned actin myofibrils and $\beta$-catenin-containing cell junctions between neonate and stem cell-derived myocytes. Ratiometric $\mathrm{Ca}^{2+}$ imaging and traction force microscopy (TFM) revealed synchronous $\mathrm{Ca}^{2+}$ transients and mechanical contractions between cells, but reduced $\mathrm{Ca}^{2+}$ levels and lower peak systolic forces were observed in $\mathrm{mES}$ - and miPS-CMs coupled with neonate myocytes. Pivotally, these differences yielded an imbalance in tension across $\mu$ tissues that was accompanied by the appearance of traction forces and substrate adhesions near the cell-cell junction. A finite element model of muscle contraction revealed that differences in isometric tension were sufficient to predict the observed pattern of adhesive forces on the substrate. Our findings suggest

(C) 2016 Aratyn-Schaus et al. This article is distributed under the terms of an AttributionNoncommercial-Share Alike-No Mirror Sites license for the first six months after the publication date (see http://www.rupress.org/terms). After six months it is available under a Creative Commons License (Attribution-Noncommercial-Share Alike 3.0 Unported license, as described at http://creativecommons.org/licenses/by-nc-sa/3.0/). 
A

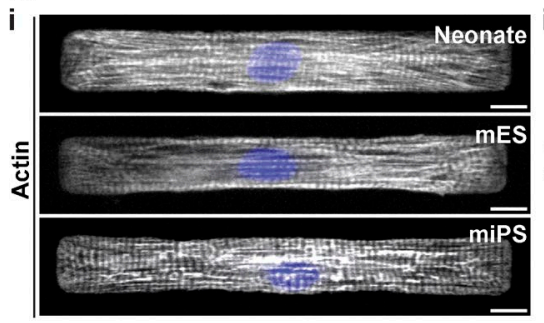

C

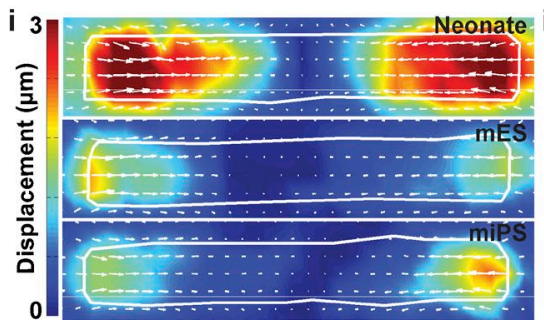

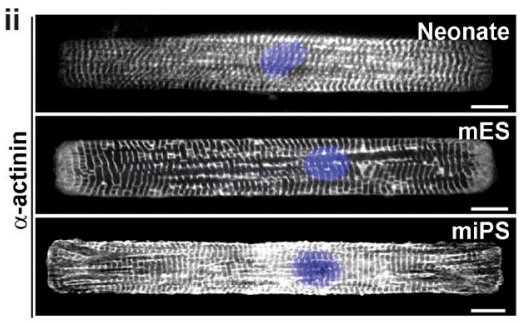

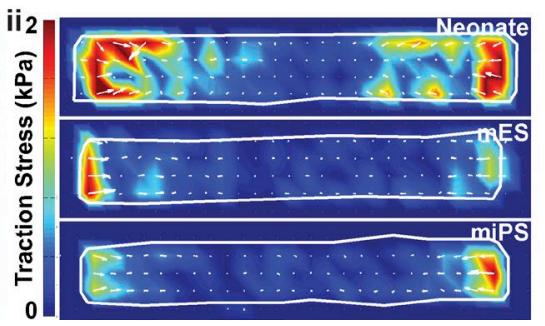

B

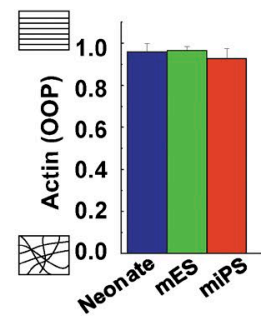

D

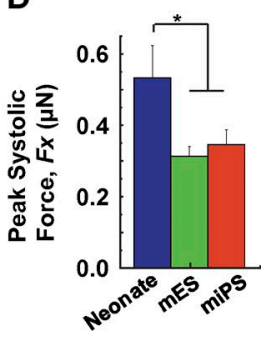

Figure 1. Isolated primary and stem cell-derived cardiac myocytes. (A) Representative images of mononucleated (DAPI, blue) mouse neonate myocytes or mES- or miPS-CMs stained for actin (i) and $\alpha$-actinin (ii). Bar, $10 \mu \mathrm{m}$. (B) Actin OOP as a function of cell type $(n=25,20$, and 9 for neonate, $\mathrm{mES}$, and miPS, respectively). (C) Representative heat maps indicating peak systolic displacement (i) and traction stress (ii). (D) Peak systolic force as a function of cell type $\left(n=8,7\right.$, and 7 for neonate, $\mathrm{mES}$, and miPS, respectively). Results presented as mean \pm SEM. ${ }^{*}, \mathrm{P}<0.05$.

that despite achieving synchronous contraction, reduced force transmission between spared and newly formed myocytes may limit repair of the contractile function in cardiac cell therapy.

\section{Results and discussion}

\section{Contractile structure and function in primary and stem cell-derived myocytes} In this study, we used myocytes harvested from neonate mouse hearts or differentiated from mES and miPS cells to model stronger native and weaker regenerated myocardium, respectively. To validate this choice, we assessed the structural and functional proficiency of isolated neonate myocytes and mESand miPS-CMs cultured on fibronectin islands (7:1 length-towidth ratio) that were microcontact-printed on soft $(13-\mathrm{kPa})$ gels that mimic the microenvironment of the healthy heart (Engler et al., 2008; McCain et al., 2014).

Neonate myocytes and mES- and miPS-CMs exhibited striated myofibrils that extended parallel to the longitudinal axis of the cell, as demonstrated by immunostains of actin (Fig. $1 \mathrm{~A}$ [i]) and $\alpha$-actinin (Fig. 1 A [ii]). To quantify actin alignment, we calculated the orientational order parameter (OOP), which yields values ranging from 0 to 1 for randomly distributed and perfectly aligned networks, respectively (Pasqualini et al., 2015). We found that both primary and stem cell-derived myocytes had highly aligned cytoskeletons, with OOP $>0.9$ (Fig. 1 B). To compare the contractile force of spontaneously beating myocytes, we used TFM. Substrates were doped with fluorescent beads whose displacement between peak systole and diastole was measured and converted into traction stress (Butler et al., 2002). Displacement (Fig. 1 C [i]) and traction stress (Fig. $1 \mathrm{C}$ [ii]) heat maps of neonate myocytes and mESand miPS-CMs revealed localization of stresses at the proximal ends of the cells, with large traction force vectors along the longitudinal axis (Videos 1-3). A comparison of peak systolic longitudinal force revealed roughly twofold lower magnitudes for mES- and miPS-CMs relative to neonate myocytes (Fig. $1 \mathrm{D}$ ).
Collectively, these results show that despite exhibiting similar cytoskeletal organization, stem cell-derived myocytes were significantly weaker than neonate myocytes.

\section{Contractile architecture in paired primary and stem cell-derived nyocytes}

For cardiac stem cell therapy to be effective, seamless integration of newly formed and existing myocardium must be achieved. This requires the formation of intercalated disks that facilitate gap junction formation and electrical conduction (Chung et al., 2007; Kim et al., 2010), as well as myofibril registration and force transmission (Parker and Ingber, 2007). We asked whether this process is impaired when cells of unequal contractile strength are coupled together. To answer this question, we engineered cardiac myocyte pairs, or $\mu$ tissues (McCain et al., 2012a,b). Homogeneous $\mu$ tissues consisted solely of pairs of neonate myocytes or mES- or miPS-CMs, and heterogeneous $\mu$ tissues were composed of a single neonate cell coupled to a $\mathrm{GFP}^{+}$mES- or miPS-CM (Fig. 2 A). We observed that myofibrils aligned parallel to the longitudinal axis and appeared registered in all pairs (Fig. 2 A). All $\mu$ tissue configurations formed cell-cell junctions and expressed $\beta$-catenin (Fig. 2 B) and connexin-43 (Fig. $2 \mathrm{C}$ ), proteins responsible for mechanical and electrical coupling, respectively. Quantitatively, myocytes spread to occupy roughly half the $\mu$ tissue area, exhibited actin OOP $>0.9$, sigmoidal junctions (McCain et al., 2012b), and elongated nuclei (Ye et al., 2014) independently of pairing type (Fig. S1). Together, these results demonstrate that in our cardiac cell therapy on-a-chip platform, heterogeneous $\mu$ tissues can exhibit the same molecular markers of engraftment that are observed in vivo.

\section{Calcium handling in paired primary and stem cell-derived myocytes}

To test the electrochemical coupling of engineered $\mu$ tissues, we quantified $\mathrm{Ca}^{2+}$ transients via dual-excitation ratiometric analysis (Lohr, 2003; Sheehy et al., 2014). Although $\mathrm{Ca}^{2+}$ transients in neonate myocytes and mES- and miPS-CMs paired in 
A

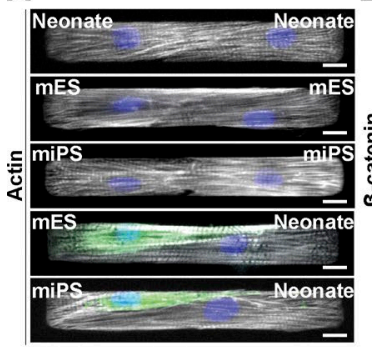

E

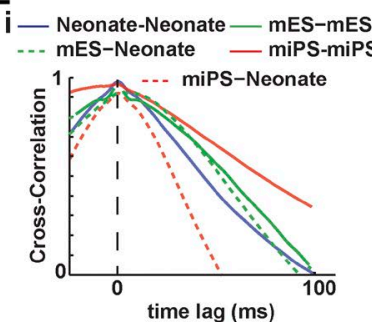

B
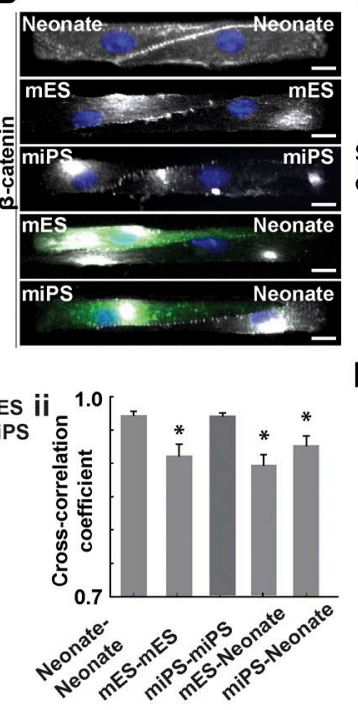

C

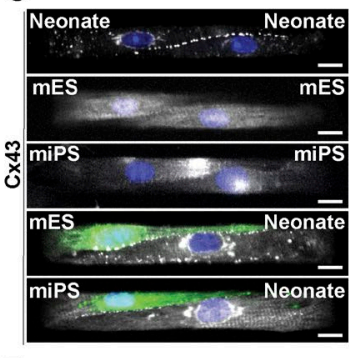

$\mathbf{F}$

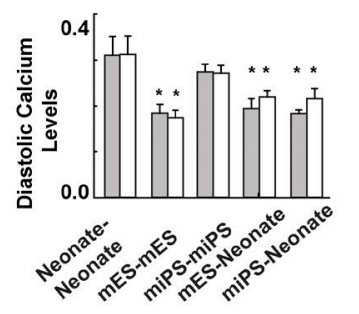

D

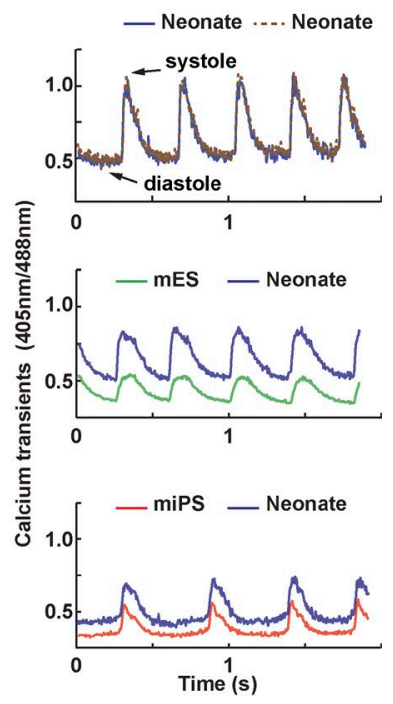

Figure 2. Structural and electrochemical coupling of paired myocytes. Representative images of pairs of mononucleated (DAPI, blue) mouse neonate myocytes or mES or miPS-CMs stained for actin (A), $\beta$-catenin (B), and connexin-43 (C). Bar, $10 \mu \mathrm{m}$. GFP-tagged (green) mES- or miPS-CMs were used for heterogeneous pairing. (D) Representative ratiometric $\mathrm{Ca}^{2+}$ transients across several contraction cycles for different ptissue types. (E) Normalized crosscorrelation functions and cross-correlation coefficients for different $\mu$ tissue types. (F) Diastolic $\mathrm{Ca}^{2+}$ levels for each cell within a cell pair. Results are presented as mean $\pm \operatorname{SEM}\left(n=8,13,9,15\right.$, and 6 for neonate-neonate, $\mathrm{mES}-\mathrm{mES}$, mES-neonate, miPS-miPS, and miPS-neonate, respectively). ${ }^{*}, \mathrm{P}<0.05$, statistically significant difference with homogeneous neonate pair.

heterogeneous pairs had different time courses, all coupled cells exhibited synchronized activity (Fig. 2 D). We constructed a cross-correlation function that measures the time lag between the two $\mathrm{Ca}^{2+}$ signals. Within the limits of our experimental sampling frequency, we observed no frame lag (Fig. 2 E [i]). This translates to a conduction velocity in excess of $5 \mathrm{~cm} / \mathrm{s}$, in good agreement with a previous study (Cohen-Karni et al., 2009). Using the cross-correlation coefficient, a metric of similarity between signals, we showed that the time course of cytoplasmic $\mathrm{Ca}^{2+}$ in homogeneous mES pairs, and all heterogeneous pairs, had significantly more variability than that of the other homogeneous pairs (Fig. $2 \mathrm{E}$ [ii]). In addition, although homogeneous pairs maintained nearly identical diastolic $\mathrm{Ca}^{2+}$ levels, mESCMs had almost twofold lower $\mathrm{Ca}^{2+}$ levels than neonate myocytes and 1.5 times lower levels than miPS-CMs. Moreover, diastolic $\mathrm{Ca}^{2+}$ levels in neonate myocytes paired with mES- or miPS-CMs were $\sim 30 \%$ lower than those observed in homogeneous neonate pairs. Correspondingly, the coupling of stem cell-derived myocytes to neonate myocytes did not increase diastolic $\mathrm{Ca}^{2+}$ levels; mES-CMs exhibited no significant change, whereas miPS-CMs experienced a significant $\sim 9 \%$ reduction compared with respective homogeneous pairings (Fig. 2 F).

Collectively, these results support the notion that myocytes at different maturation stages can form synchronized $\mu$ tissues, yet possible cell line-specific proarrhythmic heterogeneities in $\mathrm{Ca}^{2+}$ handling persist and may contribute to depressed contractile function, perhaps through a classic inotropic effect (Bers, 2001).

\section{Contractile function in paired primary and stem cell-derived myocytes}

To test whether intrinsic differences in force generation capacity between primary and stem cell-derived myocytes impact $\mu$ tissue function, we adopted TFM to study spontaneously beating cell pairs (Videos 4-6). A comparison of substrate displacement (Fig. 3 A [i]) and traction stress (Fig. 3 A [ii]) across homogeneous $\mu$ tissues revealed that paired neonatal myocytes exerted greater peak systolic displacement and traction stress than either mES- or miPS-CM $\mu$ tissues. Importantly, traction stresses adjacent to the cell-cell junctions were evident in heterogeneous pairs but virtually absent in homogeneous ones. Confocal imaging of a focal adhesion marker, vinculin, revealed focal adhesion formation at the lateral ends of all cell-pair types and occasional localization of adhesions near heterogeneous cell-cell junctions, consistent with regions of high traction stress (Fig. 3 B).

To assess whether neighboring neonate myocytes or mESor miPS-CMs exhibit mechanical synchrony when coupled, we quantified the total longitudinal traction force exerted on the substrate in each cell and at the junction during a contraction cycle (Fig. $3 \mathrm{C}$ [i]). Both mES- and miPS-CMs remained weaker than neonate myocytes in homogeneous pairs but exhibited synchronous contraction profiles and minimal junctional traction force (Fig. $3 \mathrm{C}$ [i]). In heterogeneous pairs, contraction profiles for neonate myocytes or mES- or miPS-CMs were also in phase (Fig. 3, A and C [iii and iv]), yet the total durations of the contraction cycles were $\sim 40 \%$ longer than in homogeneous neonate pairs (Fig. 3 D). Furthermore, in homogeneous pairs, neighboring cells exerted similar levels of force on the substrate: primary myocytes exerted significantly higher levels of force $(\sim 0.55 \mu \mathrm{N}$; Fig. $3 \mathrm{E})$ than mES- and miPS-CMs ( $\sim 0.25$ and $\sim 0.30 \mu \mathrm{N}$, respectively). In contrast, the difference in forces between neighboring cells within heterogeneous pairs comprised 30-35\% of the total peak systolic force exerted by the cell pair. Interestingly, traction forces at the junction between cells with uneven contractile strength were even higher on microenvironments that mimic disease states (Fig. S2), consistent with a previous study (McCain et al., 2012b). Finally, we asked whether the variability in tension generated by cell pairs resulted from differences in the performance of each cell within the pair, which might be actively contracting or 
A

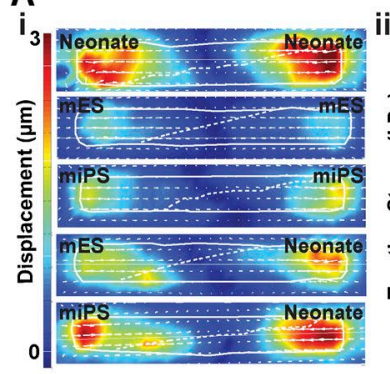

D

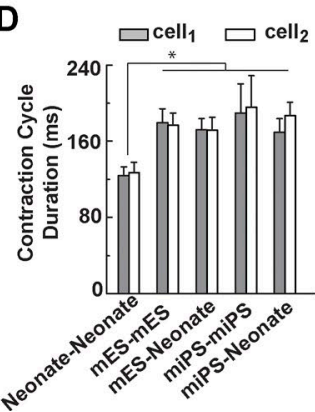

E
B
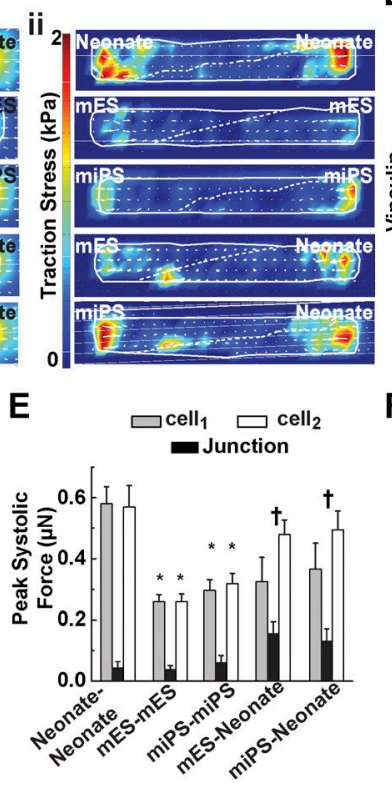

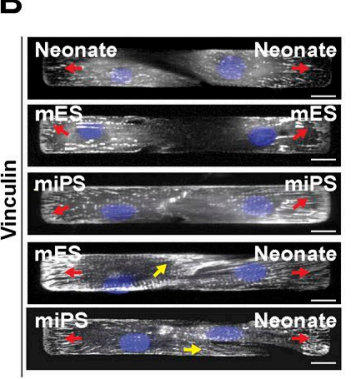

$\mathbf{F}$

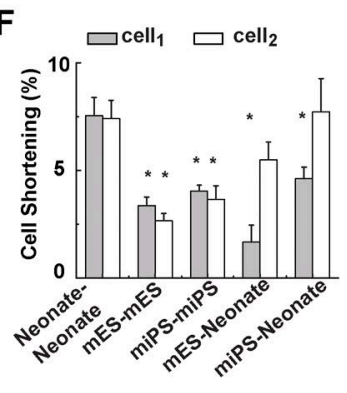

C
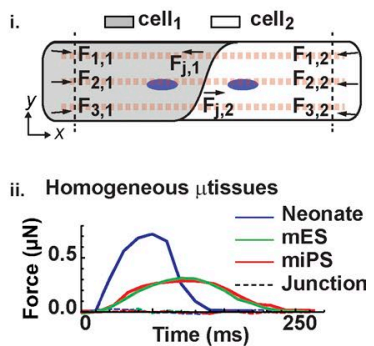

iii. mES-Neonate

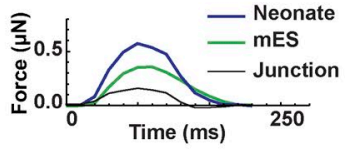

iv. MiPS-Neonate

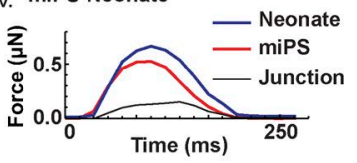

Figure 3. Mechanical coupling of paired myocytes. (A and B) Representative heat maps indicating peak systolic displacement (A, i), traction stress (A, ii), and vinculin-positive (B, gray) adhesions (arrows) for pairs of mononucleated (DAPI, blue) myocytes. Bar, $10 \mu \mathrm{m}$. (C) Schematic diagram indicating force calculations for different locations within cell pairs (i). Representative contraction cycles for each cell within homogeneous (ii) and heterogeneous mES-CM (iii) or miPS-CM (iv) pairs. (D) Duration of the contraction cycle as a function of $\mu$ tissue type. (E) Peak systolic force in different regions within cell pairs. (F) Percentage cell shortening across homogeneous and heterogeneous cell pairs. Results presented as mean $\pm \operatorname{SEM}(n=7,12,8,7$, and 7 for neonate-neonate, $\mathrm{mES}$-mES, mES-neonate, miPS-miPS, and miPS-neonate, respectively). ${ }^{*}, \mathrm{P}<0.05$, statistically significant differences from the neonate-neonate case for cellular values; ${ }^{\dagger}, \mathrm{P}<0.05$, statistically significant differences from the neonate-neonate case for junctional values.

passively stretching. In homogeneous pairs of primary myocytes, each cell within a pair shortened by roughly $7 \%$ (Fig. $3 \mathrm{~F}$ ), significantly more than in the stem cell-based homogeneous pairs (3\% and $4 \%$ for mES- and miPS-CMs, respectively). In heterogeneous pairs, mES- and miPS-CMs shortened by $1.6 \%$ and $4.6 \%$, respectively, whereas neonate myocytes within these pairs shortened by $5.6 \%$ and $7.7 \%$, respectively. These values resulted predominantly from movement by lateral edges in the direction of the neighboring cell, suggesting that both myocytes were actively contracting and not passively stretching.

Similar to what we observed in in vitro models of cardiac development and disease (McCain et al., 2012b), these results suggest that the cell-matrix adhesions located at the cell-cell junctions may have the functional role of dissipating excess force onto the substrate, thereby preventing mechanical disruption of the cell-cell junction.

\section{A computational model of paired primary and stem cell-derived myocyte mechanics} We previously documented that multiple transcriptional and functional differences exist between these primary and stem cell-derived myocyte populations, including expression of atrial transcription factors, fetal contractile protein isoforms, and reduced calcium currents (Sheehy et al., 2014). Because the relative importance of each mechanism may vary in newly formed myocytes from different species, origins, and/or repair mechanisms, we developed a generalized computational model of $\mu$ tissue mechanics (Fig. 4 A). Through this model, we directly tested whether differences in contractile strength may be responsible for the pattern of adhesion formation and traction stress observed in our study. We treated the $\mu$ tissue as a 2D continuum whose contractile behavior is governed by the Hill equation and whose anchoring is mediated by elastic constraints. Additionally, we included positive feedback between adhesion maturation and traction stress (Grosberg et al., 2011). We assigned model parameters based on literature values and our single-cell data, exclusively. Specifically, we assigned smaller magnitudes of isometric tension and strain rate constants to stem cellderived myocytes in heterogeneous pairs, because of the known inotropic effect of diastolic calcium levels (Bers, 2001). Importantly, we did not specify the locations of adhesion formation but allowed for adhesion maturation and strengthening to develop in the model (Fig. S3 and Videos 7-10).

Our simulations showed that in homogeneous $\mu$ tissues, high traction stress was distributed solely near the two lateral ends of the islands. In heterogeneous $\mu$ tissues, additional high traction stress localized to the tip of the neonate myocyte near the cell-cell interface (Fig. 4 B, red arrow). These results were consistent with our experimental traction stress maps (Fig. $3 \mathrm{~A}$ ), suggesting that the presence of a weaker cell in the pair was sufficient to cause the patterns of traction stress observed in heterogeneous cell pairs. Further, as shown in Fig. $4 \mathrm{C}$, the predicted cellular tension at peak systolic contraction was distributed continuously across the cell-cell interface for the homogeneous pair. In contrast, in the heterogeneous pair, cellular stress was lower in the stem cell-derived myocyte than in the neonate one, and a discontinuity of cellular stress occurred at the cell-cell interface. Moreover, predicted forces and the mean percent shortening (Fig. 4 D) were also in agreement with our in vitro data (Fig. 3, E and F), confirming that the stem cellderived myocytes shortened less than the neonate myocytes, yet neither cell lengthened.

Thus, our computational model suggests that pairing myocytes with intrinsically different contractile properties, such as 
A

Finite element mesh of the $\mu$ tissue

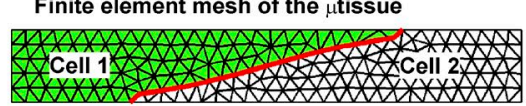

B

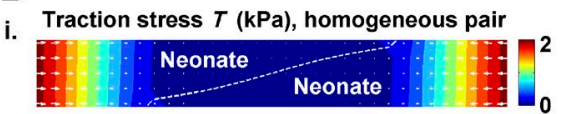

ii.

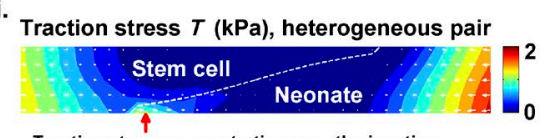

Traction stress concentration near the junction

C

i. Cellular tension (kPa), homogeneous pair

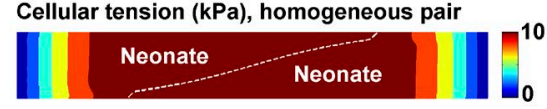

ii.

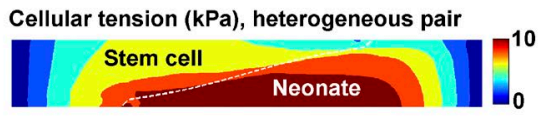

D

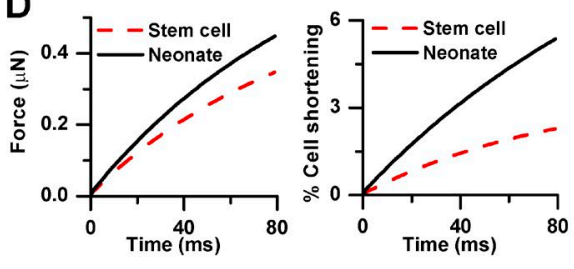

E

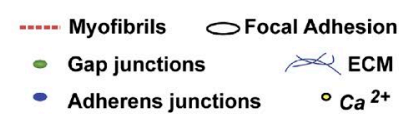

i.

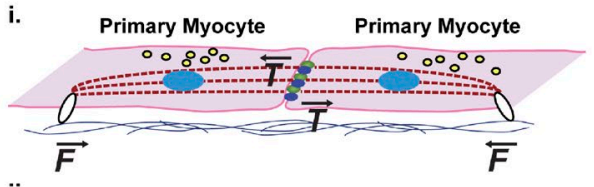

ii.

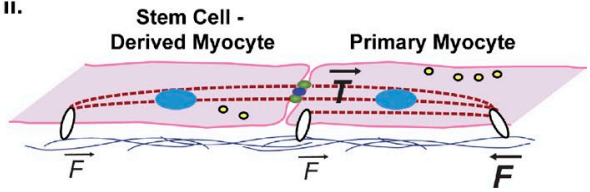

Figure 4. Computational model of $\mu$ tissue mechanics. (A) Representative finite element mesh of $\mu$ tissue (cell-cell junction highlighted in red). ( $B$ and $C$ ) Predicted patterns of local traction (B) and cellular (C) stress at peak systole for homogeneous (i) and heterogeneous (ii) pairs. The red arrow indicates high traction stress beneath the neonate myocyte near the cell-cell junction. (D) Predicted traction force and percentage shortening in heterogeneous cell pairs. (E) Schematic illustration indicates typical tension experienced at substrate adhesions within pairs of neonate cells (i) and heterogeneous (ii) ptissues. a reduction in isometric tension, is sufficient to induce force transmission remodeling at the cell-cell junction.

\section{Mechanotransduction and cardiac stem cell therapy}

We presented a novel muscle-on-a-chip platform to quantify the structural and functional engraftment of primary and stem cell-derived myocytes. We showed that weaker mES- or miPSCMs can align myofibrils, form gap junctions, and contract synchronously with stronger neonate myocytes. Further, we showed that stem cell-derived myocytes exhibit impaired calcium handling and exert lower contractile force than primary cells, resulting in a net junctional force that was balanced by compensatory cell substrate adhesions at the cell-cell junction. Our in vitro and in silico results suggest that myocytes sense the force imbalance across the cell-cell junction and respond by depositing junctional substrate adhesions that dissipate the excess force. Further studies are required to clarify whether these cell substrate adhesions are mature focal adhesions that participate in intracellular signaling. At this point, we note that in the absence of this mechanotransductive mechanism (Fig. 4 E), the cell-cell interface could be mechanically disrupted, which would compromise tissue morphogenesis and the recruitment of cell-cell coupling proteins into the nascent intercalated disk (McCain et al., 2012b).

We believe that our findings in a mouse model can be extrapolated to human cells in the context of cardiac cell therapy, even if we could not directly test our system with rare human primary myocytes that dedifferentiate rapidly in culture (Kubin et al., 2011; Morrisey, 2011). In fact, despite a variety of proposed cell types and repair mechanisms, newly formed myocytes were consistently small and mononucleated and characterized by an immature organization of sarcomeres, mitochondria, and T-tubules. Together, these characteristics suggest that newly formed myocytes may be weaker than spared ones in vivo and therefore may be subject to the compensatory mechanism we describe herein. In this context, our data suggest that stem cell-derived myocytes can form a syncytium with the native myocardium, which may explain the reduction in infarct size reported in many studies (Abdel-Latif et al., 2007; Bolli et al., 2011; Donndorf et al., 2011; Makkar et al., 2012; Traverse et al., 2012). At the same time, the heterogeneity in calcium handling and force generation properties observed between stem cell-derived and primary myocytes may result in force transmission impairment and possibly proarrhythmic events that ultimately limit the therapeutic benefit (Makkar et al., 2012; Traverse et al., 2012).

In conclusion, as protocols for the differentiation of more mature, chamber-specific human ES-/iPS-CMs become available, we envision expanding the cardiac cell therapy on-a-chip platform into a screening assay to identify cell types or pharmacological and/or gene therapy strategies that potentiate (a) the mechanical coupling between stronger and weaker myocytes in vitro and (b) the engraftment of newly generated myocytes in the spared myocardium in vivo.

\section{Materials and methods}

\section{Cell culture}

Cor.At mES- and miPS-derived cardiac myocytes. Cor.At mES-derived cardiac myocytes (lot CS25CL_V_SN_1M, production date 2/16/2010; lot CS148P_1M, production date 7/9/2010; and lot CS152_1M, production date 8/16/2010) and miPS-derived cardiac myocytes (lot CS4i4M [AXIO0013_1VL] and lot CS07CL-i, production date 5/9/2011) were cultured using medium prepared according to manufacturer specifications (Axiogenesis). In brief, cells were cultured in culture flasks precoated with $10 \mu \mathrm{g} / \mathrm{ml}$ fibronectin in puromycin-containing culture medium at $37^{\circ} \mathrm{C}$ and $5 \% \mathrm{CO}_{2}$ for $24 \mathrm{~h}$. After $48 \mathrm{~h}$, the cells were rinsed with fresh medium, without the puromycin antibiotic selection agent.

Harvest of neonatal mouse cardiomyocytes. Ventricular tissue was excised from 2-d-old neonatal BALB/c mice and dissociated into a $95 \%$ pure preparation of isolated cardiac myocytes as previously described (Sheehy et al., 2014). Excised ventricular tissue was rinsed with Hanks balanced salt solution, incubated in a $0.1 \%$ (wt/vol) solution of trypsin (USB) with agitation overnight at $4^{\circ} \mathrm{C}$, and dissociated via serial exposure to a $0.1 \%(\mathrm{wt} / \mathrm{vol})$ solution of collagenase type II (Worthington 
Biochemical) at $37^{\circ} \mathrm{C}$ for $2 \mathrm{~min}$. A 45 -min preplating step was used to reduce the number of nonmyocytes in the preparation. All procedures were approved by the Harvard Animal Care and Use Committee.

Primary and stem cell-derived cardiomyocyte seeding procedures. Cor.At mES- and miPS-derived cardiac myocytes were cultured according to manufacturer specifications (Axiogenesis) in positive selection medium containing puromycin for $48 \mathrm{~h}$ before cell dissociation by $0.25 \%$ trypsin. For heterogeneous cultures, Cor.At mES- or miPS-CMs were seeded simultaneously with isolated neonatal mouse ventricular myocytes onto microcontact-printed substrates at equal densities $\left(8,000\right.$ cells $/ \mathrm{cm}^{2}$ per cell type) and cultured in Cor.At proprietary medium (Axiogenesis). For homogeneous cultures, primary myocytes or mES-/miPS-CMs were seeded at densities of 16,000 cells/ $\mathrm{cm}^{2}$. Myocytes were cultured on microcontact-printed substrates for $3 \mathrm{~d}$ before experimentation. Experiments were performed in an incubation chamber on a laser scanning confocal microscope (Carl Zeiss) maintained at $37^{\circ} \mathrm{C}$ and cultured in Tyrode's solution $\left(1.8 \mathrm{mM} \mathrm{CaCl}_{2}, 5 \mathrm{mM}\right.$ glucose, $5 \mathrm{mM}$ Hepes, $1 \mathrm{mM} \mathrm{MgCl} 2,5.4 \mathrm{mM} \mathrm{KCl}, 135 \mathrm{mM} \mathrm{NaCl}$, and $\left.0.33 \mathrm{mM} \mathrm{NaH}_{2} \mathrm{PO}_{4}, \mathrm{pH} 7.4\right)$.

\section{Photolithography and microcontact printing}

Polymer stamps designed for microcontact printing were prepared as previously described (McCain et al., 2012b, 2014). Photolithographic masks were designed in AutoCAD (Autodesk), and consisted of rectangles of 2,000- $\mu \mathrm{m}^{2}$ surface area, spaced by $100 \mu \mathrm{m}$ on each side, with length-to-width ratios of 7:1. Silicon wafers (Wafer World) were spin-coated with SU-8 2002 photoresist (MicroChem), exposed to UV light, and submerged in propylene glycol methyl ether acetate to dissolve masked regions. Polydimethylsiloxane (Sylgard 184; Dow Corning) elastomer was poured onto the wafers, cured, and peeled to generate stamps.

\section{Fabrication and microcontact printing of polyacrylamide gels}

All functional experiments were conducted using 13 - or $90-\mathrm{kPa}$ polyacrylamide gel substrates (5\% acrylamide: $0.3 \%$ bisacrylamide; Molecular Probes) that were fabricated on $25-\mathrm{mm}$ coverslips, as previously described (McCain et al., 2012b, 2014; Ye et al., 2014). For TFM experiments, gels were doped with streptavidin-acrylamide and 200-nm fluorescent beads (Invitrogen) with a final ratio of 1:5 and 1:30 by volume, respectively. For all other experiments, gels were bead-free. Fibronectin (BD Biosciences) was biotinylated by cross-linking with biotin using Sulfo-NHS-LC-Biotin (Pierce). Polydimethylsiloxane stamps were incubated with $200 \mu \mathrm{g} / \mathrm{ml}$ of biotinylated fibronectin for $1 \mathrm{~h}$ and dried before stamping the top surface of polyacrylamide gels.

\section{Immunofluorescence}

Myocytes were fixed in 4\% PFA in PBS buffer at RT for $10 \mathrm{~min}$. Cells were immunostained by incubating for $1 \mathrm{~h}$ at RT with combinations of primary antibodies against connexin-43 (MAB3068; Millipore), $\beta$-catenin (C2206; Sigma-Aldrich), sarcomeric $\alpha$-actinin (clone EA-53; Sigma-Aldrich), vinculin (hVIN-1; Sigma-Aldrich), DAPI (Invitrogen), and Alexa Fluor 488-, 546-, or 633-conjugated phalloidin (Invitrogen). Secondary antibodies conjugated to Alexa Fluor 488, 546, or 633 (Invitrogen) were used against the appropriate primary antibody for 1-h incubation at RT. All antibodies were used at a dilution of 1:200. Immunostained samples were imaged with a confocal microscope (LSM5 LIVE; Carl Zeiss).

\section{TFM}

Spontaneously beating cardiac myocytes along with 200-nm fluorescent beads at the top surface of polyacrylamide gels were imaged sequentially at $60 \mathrm{~Hz}$ using a scanning confocal microscope, with a
$40 \times$ Plan-Apochromat oil objective. Cells were imaged at $37^{\circ} \mathrm{C}$ for at least four contraction cycles. High-resolution TFM Fourier transform traction cytometry methods were used to track bead displacement over time and determine traction stress as previously described (Butler et al., 2002). The total traction force, $F_{x}$, exerted by a cell on the substrate along the $x$ axis was calculated by summing the magnitudes of all traction force vectors, $\vec{F}_{x, i}$ and multiplying by a factor of one half:

$$
F=1 / 2\left(\left|\sum_{i} F_{L, x, i}\right|+\left|\sum_{i} F_{R, x, i}\right|\right) .
$$

\section{Ratiometric $\mathrm{Ca}^{2+}$ experiments and analysis}

Myocytes were incubated with in an $8-\mu \mathrm{M}$ solution of acetoxymethyl Fura Red (F-3021; Invitrogen) reconstituted in Pluronic F-127 (20\% solution in DMSO; P-3000MP; Invitrogen) for $20 \mathrm{~min}$ before rinses in Tyrode's solution and experimentation. Spontaneously beating cardiac myocytes were imaged sequentially at $216.5 \mathrm{~Hz}$ using a scanning confocal microscope with a $40 \times$ Plan-Apochromat oil objective at $37^{\circ} \mathrm{C}$. Images were collected at 405 and $488 \mathrm{~nm}$ and preprocessed using Image J software to correct for background illumination and outline each cell within a pair (GFP positive $\mathrm{mES}$ - and miPS-CMs were distinguishable from neonate myocytes). The mean intensity over time in each region of interest was measured and analyzed in MATLAB. Region-of-interest measurements were parsed to isolate the time course of the $\mathrm{Ca}^{2+}$ transients, as measured under 405-nm (Ca405) and 488$\mathrm{nm}(\mathrm{Ca} 488)$ illumination and used to calculate the ratio Ca405/Ca488. Only traces pertaining to cells beating at a frequency comparable with those observed during TFM experiments $(2.5 \pm 0.8 \mathrm{~Hz})$ were included in this phase. Four to six steady-state transients were averaged in each recording and used to calculate diastolic level, peak level, time to peak, and duration of the $\mathrm{Ca}^{2+}$ transient at $50 \%$ and $90 \%$ decay.

We performed two separate analyses of the ratiometric recording. First, the trains of calcium transients recorded in each cell in the pair were subjected to cross-correlation analysis (Cohen-Karni et al., 2009). In brief, the normalized cross-correlation of two signals $x$ and $y$ was calculated with the following formula:

$$
r(\tau)=\frac{1}{N} \frac{\sum_{i=1}^{N}\left[\left(x_{i}-\mu_{x}\right)\left(y_{i-\tau}-\mu_{y}\right)\right]}{\sigma_{x} \sigma_{y}}
$$

where $i=\{1,2, \ldots, N\}$ are the sampled values of the signals; $\mu_{x}, \mu_{y}, \sigma_{x}$, and $\sigma_{y}$ are the means and SDs of $x$ and $y$, respectively, and $\tau$ represents a time lag. The value of $\tau$ for which $r$ is maximum is an estimate of the conduction velocity, as it determines how many frames it takes for the peak of one signal to travel to the second one. The maximum value of $r(\tau)$ (or the cross-correlation coefficient) provides an estimate of how globally similar the two signals are.

\section{Statistical analysis}

Statistical analysis was conducted with SigmaPlot; data were first tested with the Shapiro-Wilk test for normality and the Levene median test for equal variance. One-way analysis of variance tests were conducted for normally distributed datasets, followed by pairwise posthoc Student-Newman-Keuls test. Alternatively, statistical conclusions were drawn using analysis of variance on ranks followed by Dunn's test for pairwise comparison.

\section{Cell mechanics model}

In our finite element model, the cell-pair $\mu$ tissue is treated as a 2D continuum (Fig. S3 A). Cell substrate adhesion is modeled by applying elastic constraints to the displacement field throughout the $\mu$ tissue domain (Fig. S3 B). Thus, the equilibrium equations of the $2 \mathrm{D} \mu$ tissue in the $(x, y)$ plane can be written as (Sadd, 2014): 


$$
\frac{\partial\left(h \sigma_{x}\right)}{\partial x}+\frac{\partial\left(h \tau_{x y}\right)}{\partial y}-k_{c s}(x, y) u_{x}=0
$$

and

$$
\frac{\partial\left(h \tau_{x y}\right)}{\partial x}+\frac{\partial\left(h \sigma_{y}\right)}{\partial y}-k_{c s}(x, y) u_{y}=0
$$

where $\sigma_{x}, \sigma_{y}$, and $\tau_{x y}$ are stress components; $u_{x}$ and $u_{y}$ are displacement components; $h$ is the height of the $\mu$ tissue; and $k_{c s}(x, y)$ is the cell substrate linkage constant. The traction stress components on the substrate can be written as

$$
T_{x}=k_{c s}(x, y) u_{x}
$$

and

$$
T_{y}=k_{c s}(x, y) u_{y}
$$

We plot the magnitude of the traction stress $T=\sqrt{T_{x}^{2}+T_{y}^{2}}$ for the traction stress maps in Fig. 4 in the text and in Fig. S3. Note that in the $2 \mathrm{D}$ problem, the cell substrate adhesion enters the equilibrium equations as "body force," instead of boundary conditions. The position-dependent cell substrate linkage constant $k_{c s}(x, y)$ is a lumped parameter that accounts for both the substrate elasticity and the density of bound integrin. Because the substrate elasticity is not varied in this work, $k_{c s}(x, y)$ is defined as a function of the density of bound integrin $\rho(x, y)$ alone:

$$
k_{c s}(x, y)=k_{\max } \frac{\rho(x, y)}{\rho_{\max }},
$$

where parameter $k_{\max }$ is the maximal cell substrate linkage stiffness, and parameter $\rho_{\max }$ is the maximal density of bound integrin. We use $k_{\max }=0.7 \mathrm{kPa} / \mu \mathrm{m}$ and $\rho_{\max }=400 \mu \mathrm{m}^{-2}$ in all simulations. The focal adhesion maturation is thought to be coupled with the traction stress with a positive feedback loop (Grosberg et al., 2011). Here, we implemented the positive feedback using the following simple form:

$$
\rho(x, y)=\frac{\rho_{\max }\left[T(x, y) / T_{0}\right]^{4}}{1+\left[T(x, y) / T_{0}\right]^{4}},
$$

where $T_{0}$ is the traction stress threshold, the reference to which the density of bound integrin is redistributed based on the magnitude of the traction stress. We use $T_{0}=1.0 \mathrm{kPa}$ for the homogeneous pair and $T_{0}=0.56 \mathrm{kPa}$ for the heterogeneous pair in the simulations. The purpose of applying Eq. 6 is to make the bound integrin density $\rho(x, y)$ approach $\rho_{\max }$ when $T(x, y)>T_{0}$ and to make it approach zero when $T(x, y)<T_{0}$.

Cell-cell adhesion is modeled as elastic springs and implemented into the finite element model by defining its deformation energy $E_{c c}$ as

$$
E_{c c}=\frac{k_{c c}}{2} \int_{\Gamma}\left[\left(u_{x}^{1}-u_{x}^{2}\right)^{2}+\left(u_{y}^{1}-u_{y}^{2}\right)^{2}\right] d s,
$$

where $k_{c c}$ is the cell-cell adhesion spring constant; the path $\Gamma$ denotes the cell-cell interface (Fig. 4 A); $S$ denotes arch length along $\Gamma$; and $\left(u_{x}^{1}, u_{y}^{1}\right)$ and $\left(u_{x}^{2}, u_{y}^{2}\right)$ are displacement components of cells 1 and 2 at the cell-cell interface, respectively. We use $k_{c c}=0.05 \mathrm{kPa}$ for the homogeneous pair and $T_{0}=0.01 \mathrm{kPa}$ for the heterogeneous pair in the simulations.

We adopted Hill's three-element model (Fung, 1993) for the contraction of cardiomyocytes. A schematic of Hill's three-element model is shown in Fig. S3 (B and C), in which the myofibril is modeled as a contractile element and an elastic spring in series, and a parallel element represents the passive components of the cytoskeleton that deforms under active contraction. The stress-strain equations of the three-element model take the following forms when the direction of the myofibril is parallel to the $x$ axis of the coordinate system:

$$
\begin{gathered}
{\left[\begin{array}{c}
\sigma_{x} \\
\sigma_{y} \\
\tau_{x y}
\end{array}\right]=\left[\begin{array}{ccc}
\frac{E}{1-v^{2}} & \frac{E v}{1-v^{2}} & 0 \\
\frac{E v}{1-v^{2}} & \frac{E}{1-v^{2}} & 0 \\
0 & 0 & \frac{E}{2(1+v)}
\end{array}\right]\left[\begin{array}{c}
\varepsilon_{x} \\
\varepsilon_{y} \\
2 \varepsilon_{x y}
\end{array}\right]+\left[\begin{array}{c}
\sigma_{c} \\
0 \\
0
\end{array}\right],} \\
\frac{\sigma_{c}}{\sigma_{c 0}}=1-\frac{1}{\gamma} \frac{d \varepsilon_{c}}{d t},
\end{gathered}
$$

and

$$
\frac{d \varepsilon_{x}}{d t}=\frac{1}{E_{s}} \frac{d \sigma_{c}}{d t}+\frac{d \varepsilon_{c}}{d t}
$$

Table 1. Summary of parameters and variables used in the finite element simulations

\begin{tabular}{lll}
\hline Parameter & \multicolumn{1}{c}{ Definition } & \multicolumn{1}{c}{ Parameter values } \\
\hline$E, \nu$ & Elastic moduli of the cell & $E=10 \mathrm{kPa}, \nu=0.49$ \\
$E s$ & Elastic modulus of the series element & $2 \mathrm{MPa}$ \\
$\sigma_{c 0}$ & Isometric tension of contraction & Neonate: $20 \mathrm{kPa} ;$ stem cell: $10 \mathrm{kPa}$ \\
$\gamma$ & Strain rate constant & Neonate: $-1.0 \mathrm{~s}^{-1} ;$ Stem cell: $-0.5 \mathrm{~s}^{-1}$ \\
$k_{\max }$ & Maximal value of cell substrate linkage constant & $0.7 \mathrm{kPa} / \mu \mathrm{m}$ \\
$k_{c s}(x, y)$ & Cell substrate linkage constant & 0.0 to $\sim 0.7 \mathrm{kPa} / \mathrm{\mu m}$ \\
$k_{c c}$ & Spring constant of cell substrate adhesion & 0.01 to $\sim 0.05 \mathrm{kPa}$ \\
$\rho_{\max }$ & Maximal density of bound integrin & $400 \mu \mathrm{m}^{-2}$ \\
$\rho(x, y)$ & Density of bound integrin & 0 to $\sim 400 \mu \mathrm{m}^{-2}$ \\
$T_{0}$ & Traction stress threshold & 0.65 to $\sim 1.0 \mathrm{kPa}$ \\
$\sigma_{c}$ & Contractile stress & $\mathrm{NA}$ \\
$\sigma_{x \prime} \sigma_{y,} \tau_{x y}$ & Components of the stress tensor & $\mathrm{NA}$ \\
$u_{x \prime} u_{y}$ & Components of the displacement vector & $\mathrm{NA}$ \\
$\varepsilon_{x \prime}, \varepsilon_{y \prime} \varepsilon_{x y}$ & Components of the strain tensor & $\mathrm{NA}$ \\
$T_{x \prime} \tau_{y}$ & Components of the traction stress & $\mathrm{NA}$ \\
\hline
\end{tabular}


where Eq. 8 represents the overall stress-strain relation of the three-element model shown in Fig. S3 (B-E); $\nu$ is the elastic modulus of the parallel element; $\varepsilon_{x}, \varepsilon_{y}$, and $\varepsilon_{x y}$ are strain components; and $\sigma_{c}$ is the contractile stress. Eq. 9 is the linearized relation for the contractile element, and $\sigma_{c 0}$ and $\gamma$ are the isometric tension and the strain rate constant of the contractile element, respectively. Eq. 10 simply represents the strain summation for the series element and contractile element, and $E_{S}$ is the elastic modulus of the series element. Note that the contractile element generates tensile stress only along the direction of myofibril. Thus, the myofibril orientation needs to be specified in the simulations. In all simulations, we assume that the myofibrils of the $\mu$ tissue align parallel to the longitudinal axis. A list of all parameters in reported in Table 1.

\section{Finite element simulations}

Eqs. 1-9 were implemented in our in-house finite element program. The cell-pair $\mu$ tissue is meshed using three-node triangular elements with mesh size of $\sim 2.0 \mu \mathrm{m}$. The total area of the $\mu$ tissue is $2,500 \mu \mathrm{m}^{2}$, and the aspect ratio is 7:1. The thickness of the $\mu$ tissue is $h=3 \mu \mathrm{m}$. The finite element domain is divided into two regions: cell 1 (green region) and cell 2 (white region), as shown in Fig. 4 A. Finite element nodes of two different cells on the cell-cell interface overlap and are connected by elastic springs derived from Eq. 7 .

Our finite element model is focused on the time period of diastolic to peak systolic contraction and is a dynamic process. An explicit time integration scheme was used to obtain time-dependent solutions of stress and strain fields from diastolic to peak systolic contraction, in which the time increment is set to be $1 \mathrm{~ms}$ and the time to peak systolic contraction is set to be $80 \mathrm{~ms}$ for all simulations. For the initial conditions of the simulations, the stress and strain components are set to be zero at time $t=0$ at the diastolic state.

We first performed simulations to determine the distribution of bound integrin in the steady state. Assuming the uniform distribution of bound integrin (i.e., $\rho(x, y)=400 \mu \mathrm{m}^{-2}$ on the entire island) at the beginning, traction stress was calculated at the peak systolic contraction. The magnitude of the traction stress $T$ was then substituted into Eq. 6 to update the distribution of bound integrin. The updated distribution of bound integrin $\rho(x, y)$ was substituted into Eq. 5 to calculate the cell substrate linkage constant $k_{c s}(x, y)$ in the steady state. In the present model, we are more concerned about the qualitative aspect of the problem; thus only one iteration was run to obtain the steady state. This procedure was performed for the homogeneous (Case 1) and heterogeneous (Case 2) cell pairs, as illustrated in Fig. S4 D. The cell substrate linkage constant $k_{c s}(x, y)$ at the steady state was then used in the new simulations in which the final traction stress, cellular tension (i.e., $\sigma_{x}$ ), and shortening were predicted, which are presented in Fig. 4 (B-D). Videos 7-10 show the time course of traction stress and cellular tension predicted in the simulations for homogeneous and heterogeneous cell pairs.

\section{Online supplemental material}

Fig. S1 shows quantitative characterization of cell morphology in homogeneous and heterogeneous $\mu$ tissues. Fig. S2 shows junctional forces in heterogeneous $\mu$ tissues in disease-mimicking microenvironments. Fig. S3 shows computational mechanics and finite element simulations. Videos 1-3 show the time course of the spontaneous contraction of individual microcontact-printed mouse neonate-, mES-, and miPS-derived cardiomyocytes. Videos 4-6 show the time course of the spontaneous contraction of a homogeneous neonate pair and heterogeneous pairs containing either mES- or miPSderived cardiomyocytes. Videos 7 and 8 show the time course of the simulated traction stress and cellular tension in a homogeneous pair. Videos 9 and 10 show the time course of the simulated traction stress and cellular tension in a heterogeneous pair. Online supplemental material is available at http://www.jcb.org/cgi/content/full/jcb.201508026/DC1.

\section{Acknowledgments}

We acknowledge the use of the Harvard Center for Nanoscale Systems micro- and nanofabrication facilities, as well as Axiogenesis for providing Cor.At murine ES- and iPS-CMs and Cor.At culture media.

The authors acknowledge funding support from the National Institutes of Health/National Center for Advancing Translational Sciences grant UH3 TR000522 "Human Cardio-Pulmonary System on a Chip," National Institutes of Health/National Heart, Lung, and Blood Institute grant UO 1 HL 100408 "Human Pluripotent Stem Cells and Progenitor Models of Cardiac and Blood Diseases," and the Harvard University Materials Research Science and Engineering Center.

The authors declare no competing financial interests.

Submitted: 7 August 2015

Accepted: 15 January 2016

\section{References}

Abdel-Latif, A., R. Bolli, I.M. Tleyjeh, V.M. Montori, E.C. Perin, C.A. Hornung, E.K. Zuba-Surma, M. Al-Mallah, and B. Dawn. 2007. Adult bone marrow-derived cells for cardiac repair: A systematic review and metaanalysis. Arch. Intern. Med. 167:989-997. http://dx.doi.org/10.1001/ archinte.167.10.989

Bers, D.M. 2001. Excitation-Contraction Coupling and Cardiac Contractile Force. Springer Netherlands, Dordrecht, Netherlands. 237 pp. http://dx .doi.org/10.1007/978-94-010-0658-3

Bers, D.M. 2002. Cardiac excitation-contraction coupling. Nature. 415:198-205. http://dx.doi.org/10.1038/415198a

Bolli, R., A.R. Chugh, D. D'Amario, J.H. Loughran, M.F. Stoddard, S. Ikram, G.M. Beache, S.G. Wagner, A. Leri, T. Hosoda, et al. 2011. Cardiac stem cells in patients with ischaemic cardiomyopathy (SCIPIO): Initial results of a randomised phase 1 trial. Lancet. 378:1847-1857. http://dx.doi.org /10.1016/S0140-6736(11)61590-0

Butler, J.P., I.M. Tolić-Nørrelykke, B. Fabry, and J.J. Fredberg. 2002. Traction fields, moments, and strain energy that cells exert on their surroundings. Am. J. Physiol. Cell Physiol. 282:C595-C605. http://dx.doi.org/10.1152 /ajpcell.00270.2001

Chung, C.Y., H. Bien, and E. Entcheva. 2007. The role of cardiac tissue alignment in modulating electrical function. J. Cardiovasc. Electrophysiol. 18:1323-1329. http://dx.doi.org/10.1111/j.1540-8167.2007.00959.x

Cohen-Karni, T., B.P. Timko, L.E. Weiss, and C.M. Lieber. 2009. Flexible electrical recording from cells using nanowire transistor arrays. Proc. Natl. Acad. Sci. USA. 106:7309-7313. http://dx.doi.org/10.1073/pnas .0902752106

Donndorf, P., G. Kundt, A. Kaminski, C. Yerebakan, A. Liebold, G. Steinhoff, and A. Glass. 2011. Intramyocardial bone marrow stem cell transplantation during coronary artery bypass surgery: A meta-analysis. J. Thorac. Cardiovasc. Surg. 142:911-920. http://dx.doi.org/10.1016/j.jtcvs.2010.12.013

Engler, A.J., C. Carag-Krieger, C.P. Johnson, M. Raab, H.Y. Tang, D.W. Speicher, J.W. Sanger, J.M. Sanger, and D.E. Discher. 2008. Embryonic cardiomyocytes beat best on a matrix with heart-like elasticity: Scar-like rigidity inhibits beating. J. Cell Sci. 121:3794-3802. http://dx.doi.org/10 $.1242 /$ jcs.029678

Fung, Y.C. 1993. Biomechanics: Mechanical Properties of Living Tissues. Springer, New York. 568 pp. http://dx.doi.org/10.1007/978-1-4757-2257-4

Grosberg, A., P.L. Kuo, C.L. Guo, N.A. Geisse, M.A. Bray, W.J. Adams, S.P. Sheehy, and K.K. Parker. 2011. Self-organization of muscle cell structure and function. PLOS Comput. Biol. 7:e1001088. http://dx.doi .org/10.1371/journal.pcbi.1001088

Hirschy, A., F. Schatzmann, E. Ehler, and J.C. Perriard. 2006. Establishment of cardiac cytoarchitecture in the developing mouse heart. Dev. Biol. 289:430-441. http://dx.doi.org/10.1016/j.ydbio.2005.10.046

Kehat, I., L. Khimovich, O. Caspi, A. Gepstein, R. Shofti, G. Arbel, I. Huber, J. Satin, J. Itskovitz-Eldor, and L. Gepstein. 2004. Electromechanical integration of cardiomyocytes derived from human embryonic stem cells. Nat. Biotechnol. 22:1282-1289. http://dx.doi.org/10.1038/nbt1014 
Kim, D.H., E.A. Lipke, P. Kim, R. Cheong, S. Thompson, M. Delannoy, K.Y. Suh, L. Tung, and A. Levchenko. 2010. Nanoscale cues regulate the structure and function of macroscopic cardiac tissue constructs. Proc. Natl. Acad. Sci. USA. 107:565-570. http://dx.doi.org/10.1073/pnas.0906504107

Kubin, T., J. Pöling, S. Kostin, P. Gajawada, S. Hein, W. Rees, A. Wietelmann, M. Tanaka, H. Lörchner, S. Schimanski, et al. 2011. Oncostatin M is a major mediator of cardiomyocyte dedifferentiation and remodeling. Cell Stem Cell. 9:420-432. http://dx.doi.org/10.1016/j.stem.2011.08.013

Laflamme, M.A., S. Zbinden, S.E. Epstein, and C.E. Murry. 2007. Cell-based therapy for myocardial ischemia and infarction: Pathophysiological mechanisms. Annu. Rev. Pathol. 2:307-339. http://dx.doi.org/10.1146/ annurev.pathol.2.010506.092038

Lohr, C. 2003. Monitoring neuronal calcium signalling using a new method for ratiometric confocal calcium imaging. Cell Calcium. 34:295-303. http:// dx.doi.org/10.1016/S0143-4160(03)00105-2

Makkar, R.R., R.R. Smith, K. Cheng, K. Malliaras, L.E. Thomson, D. Berman, L.S. Czer, L. Marbán, A. Mendizabal, P.V. Johnston, et al. 2012. Intracoronary cardiosphere-derived cells for heart regeneration after myocardial infarction (CADUCEUS): A prospective, randomised phase 1 trial. Lancet. 379:895-904. http://dx.doi.org/10.1016/S0140 $-6736(12) 60195-0$

McCain, M.L., T. Desplantez, N.A. Geisse, B. Rothen-Rutishauser, H. Oberer, K.K. Parker, and A.G. Kleber. 2012a. Cell-to-cell coupling in engineered pairs of rat ventricular cardiomyocytes: Relation between $\mathrm{Cx} 43$ immunofluorescence and intercellular electrical conductance. Am. J. Physiol. Heart Circ. Physiol. 302:H443-H450. http://dx.doi.org/10 .1152/ajpheart.01218.2010

McCain, M.L., H. Lee, Y. Aratyn-Schaus, A.G. Kléber, and K.K. Parker. 2012b. Cooperative coupling of cell-matrix and cell-cell adhesions in cardiac muscle. Proc. Natl. Acad. Sci. USA. 109:9881-9886. http://dx.doi.org/10 $.1073 /$ pnas.1203007109

McCain, M.L., H. Yuan, F.S. Pasqualini, P.H. Campbell, and K.K. Parker. 2014. Matrix elasticity regulates the optimal cardiac myocyte shape for contractility. Am. J. Physiol. Heart Circ. Physiol. 306:H1525-H1539. http://dx.doi.org/10.1152/ajpheart.00799.2013

Menasché, P., A.A. Hagège, M. Scorsin, B. Pouzet, M. Desnos, D. Duboc, K. Schwartz, J.T. Vilquin, and J.P. Marolleau. 2001. Myoblast transplantation for heart failure. Lancet. 357:279-280. http://dx.doi.org /10.1016/S0140-6736(00)03617-5

Morrisey, E.E. 2011. Rewind to recover: Dedifferentiation after cardiac injury. Cell Stem Cell. 9:387-388. http://dx.doi.org/10.1016/j.stem.2011.10.011

Parker, K.K., and D.E. Ingber. 2007. Extracellular matrix, mechanotransduction and structural hierarchies in heart tissue engineering. Philos. Trans. R. Soc. Lond. B Biol. Sci. 362:1267-1279. http://dx.doi.org/10.1098/rstb.2007.2114

Pasqualini, F.S., S.P. Sheehy, A. Agarwal, Y. Aratyn-Schaus, and K.K. Parker. 2015. Structural phenotyping of stem cell-derived cardiomyocytes. Stem Cell Rep. 4:340-347. http://dx.doi.org/10.1016/j.stemcr.2015.01.020

Sadd, M.H. 2014. Elasticity. Third edition. In Theory, Applications, and Numerics. Academic Press, Cambridge, MA. 600 pp.

Sheehy, S.P., F. Pasqualini, A. Grosberg, S.J. Park, Y. Aratyn-Schaus, and K.K. Parker. 2014. Quality metrics for stem cell-derived cardiac myocytes. Stem Cell Rep. 2:282-294. http://dx.doi.org/10.1016/j.stemcr.2014.01.015

Shiba, Y., S. Fernandes, W.Z. Zhu, D. Filice, V. Muskheli, J. Kim, N.J. Palpant, J. Gantz, K.W. Moyes, H. Reinecke, et al. 2012. Human ES-cell-derived cardiomyocytes electrically couple and suppress arrhythmias in injured hearts. Nature. 489:322-325. http://dx.doi.org/10.1038/nature11317

Strauer, B.E., M. Brehm, T. Zeus, M. Köstering, A. Hernandez, R.V. Sorg, G. Kögler, and P. Wernet. 2002. Repair of infarcted myocardium by autologous intracoronary mononuclear bone marrow cell transplantation in humans. Circulation. 106:1913-1918. http://dx.doi.org/10.1161/01 .CIR.0000034046.87607.1C

Traverse, J.H., T.D. Henry, C.J. Pepine, J.T. Willerson, D.X. Zhao, S.G. Ellis, J.R. Forder, R.D. Anderson, A.K. Hatzopoulos, M.S. Penn, et al. 2012. Effect of the use and timing of bone marrow mononuclear cell delivery on left ventricular function after acute myocardial infarction: The TIME randomized trial. JAMA. 308:2380-2389. http://dx.doi.org/10.1001/jama.2012.28726

Wu, J.C., H.C. Sung, T.H. Chung, and R.M. DePhilip. 2002. Role of N-cadherinand integrin-based costameres in the development of rat cardiomyocytes. J. Cell. Biochem. 84:717-724. http://dx.doi.org/10.1002/jcb.10092

Ye, G.J., Y. Aratyn-Schaus, A.P. Nesmith, F.S. Pasqualini, P.W. Alford, and K.K. Parker. 2014. The contractile strength of vascular smooth muscle myocytes is shape dependent. Integr Biol (Camb). 6:152-163. http://dx .doi.org/10.1039/c3ib40230d 